\title{
Approximate controllability of fractional integro-differential equations involving nonlocal initial conditions
}

\author{
NI Mahmudov ${ }^{*}$ and S Zorlu
}

\section{"Correspondence:}

nazim.mahmudov@emu.edu.tr Eastern Mediterranean University, via Mersin 10, Gazimagusa, T.R.

North Cyprus, Turkey

\begin{abstract}
We discuss the approximate controllability of nonlinear fractional integro-differential system under the assumptions that the corresponding linear system is approximately controllable. Using the fixed-point technique, fractional calculus and methods of controllability theory, a new set of sufficient conditions for approximate controllability of fractional integro-differential equations are formulated and proved. The results in this paper are generalization and continuation of the recent results on this issue. An example is provided to show the application of our result.
\end{abstract}

\section{Introduction}

Controllability is one of the fundamental concepts in mathematical control theory, which plays an important role in control systems. The controllability of nonlinear systems represented by evolution equations or inclusions in abstract spaces and qualitative theory of fractional differential equations has been extensively studied by several authors. An extensive list of these publications can be found in [1-44] and the references therein. Recently, the approximate controllability for various kinds of (fractional) differential equations has generated considerable interest. A pioneering work on the approximate controllability of deterministic and stochastic systems has been reported by Bashirov and Mahmudov [5], Dauer and Mahmudov [8] and Mahmudov [10]. Sakthivel et al. [28] studied the approximate controllability of nonlinear deterministic and stochastic evolution systems with unbounded delay in abstract spaces. On the other hand, the fractional differential equation has gained more attention due to its demonstrated applications in numerous seemingly diverse and widespread fields of science and engineering. Yan [45] derived a set of sufficient conditions for the controllability of fractional-order partial neutral functional integrodifferential inclusions with infinite delay in Banach spaces. Debbouche and Baleanu [1] established the controllability result for a class of fractional evolution nonlocal impulsive quasi-linear delay integro-differential systems in a Banach space using the theory of fractional calculus and fixed point technique. However, there exists only a limited number of papers on the approximate controllability of the fractional nonlinear evolution systems. Sakthivel et al. [28] studied the approximate controllability of deterministic semilinear fractional differential equations in Hilbert spaces. Wang [40] investigated the nonlocal controllability of fractional evolution systems. Surendra Kumar and Sukavanam [33] obtained a new set of sufficient conditions for the approximate controllability of a class of 
semilinear delay control systems of fractional order using the contraction principle and the Schauder fixed-point theorem. More recently, Sakthivel et al. [27] derived a new set of sufficient conditions for approximate controllability of fractional stochastic differential equations.

In this paper, we discuss the approximate controllability of nonlinear fractional integrodifferential system under the assumption that the corresponding linear system is approximately controllable. We consider the following fractional integro-differential control system involving nonlocal conditions,

$$
\begin{aligned}
& { }^{C} D_{t}^{\beta} x(t)=-A x(t)+f(t, x(t))+\int_{0}^{t} K(t-s) g(s, x(s)) d s+B u(t), \\
& x(0)=x_{0}+h(x),
\end{aligned}
$$

in $X_{\alpha}$, where ${ }^{C} D_{t}^{\beta}, 0<\beta<1$, stands for the Caputo fractional derivative of order $\beta$, and $f:[0, T] \times X_{\alpha} \rightarrow X, g:[0, T] \times X_{\alpha} \rightarrow X, K:[0, T] \rightarrow R^{+}, h: C\left([0, T] ; X_{\alpha}\right) \rightarrow X_{\alpha}$ are given functions to be specified later. Here, $(-A, D(A))$ is the infinitesimal generator of a compact analytic semigroup of bounded linear operators $S(t), t \geq 0$, on a real Hilbert space $X . B$ is a linear bounded operator from a real Hilbert space $U$ to $X$.

The rest of this paper is organized as follows. In Section 2, we give some preliminary results on the fractional powers of the generator of an analytic compact semigroup and introduce the mild solution of system (1). In Section 3, we study the existence of mild solutions for system (1) under the feedback control $u_{\varepsilon}(t, x)$ defined in (5). We show that the control system (1) is approximately controllable on $[0, T]$ provided that the corresponding linear system is approximately controllable. Finally, an example is given to demonstrate the applicability of our result.

\section{Preliminaries}

In this section, we introduce some facts about the fractional powers of the generator of a compact analytic semigroup, the Caputo fractional derivative that are used throughout this paper.

We assume that $X$ is a Hilbert space with norm $\|\cdot\|:=\sqrt{\langle\cdot, \cdot\rangle}$. Let $C([0, T], X)$ be the Banach space of continuous functions from $[0, T]$ into $X$ with the norm $\|x\|=$ $\sup _{t \in[0, T]}\|x(t)\|$, here $x \in C([0, T], X)$. In this paper, we also assume that $-A: D(A) \subset X \rightarrow$ $X$ is the infinitesimal generator of a compact analytic semigroup $S(t), t>0$, of uniformly bounded linear operator in $X$, that is, there exists $M>1$ such that $\|S(t)\| \leq M$ for all $t \geq 0$. Without loss of generality, let $0 \in \rho(A)$, where $\rho(A)$ is the resolvent set of $A$. Then for any $\alpha>0$, we can define $A^{-\alpha}$ by

$$
A^{-\alpha}:=\frac{1}{\Gamma(\alpha)} \int_{0}^{\infty} t^{\alpha-1} S(t) d t
$$

It follows that each $A^{-\alpha}$ is an injective continuous endomorphism of $X$. Hence we can define $A^{\alpha}:=\left(A^{-\alpha}\right)^{-1}$, which is a closed bijective linear operator in $X$. It can be shown that each $A^{\alpha}$ has dense domain and that $D\left(A^{\beta}\right) \subset D\left(A^{\alpha}\right)$ for $0 \leq \alpha \leq \beta$. Moreover, $A^{\alpha+\beta} x=$ $A^{\alpha} A^{\beta} x=A^{\beta} A^{\alpha} x$ for every $\alpha, \beta \in R$ and $x \in D\left(A^{\mu}\right)$ with $\mu:=\max (\alpha, \beta, \alpha+\beta)$, where $A^{0}=I$, $I$ is the identity in $X$. (For proofs of these facts, we refer to the literature $[15,20,22]$.) 
We denote by $X_{\alpha}$ the Hilbert space of $D\left(A^{\alpha}\right)$ equipped with norm $\|x\|_{\alpha}:=\left\|A^{\alpha} x\right\|=$ $\sqrt{\left\langle A^{\alpha} x, A^{\alpha} x\right\rangle}$ for $x \in D\left(A^{\alpha}\right)$, which is equivalent to the graph norm of $A^{\alpha}$. Then we have $X_{\beta} \hookrightarrow X_{\alpha}$, for $0 \leq \alpha \leq \beta$ (with $X_{0}=X$ ) and the embedding is continuous. Moreover, $A^{\alpha}$ has the following basic properties.

Lemma 1 [42] $A^{\alpha}$ and $S(t)$ have the following properties.

(i) $S(t): X \rightarrow X_{\alpha}$ for each $t>0$ and $\alpha \geq 0$.

(ii) $A^{\alpha} S(t) x=S(t) A^{\alpha} x$ for each $x \in D\left(A^{\alpha}\right)$ and $t \geq 0$.

(iii) For every $t>0, A^{\alpha} S(t)$ is bounded in $X$ and there exists $M_{\alpha}>0$ such that

$$
\left\|A^{\alpha} S(t)\right\| \leq M_{\alpha} t^{-\alpha}
$$

(iv) $A^{-\alpha}$ is a bounded linear operator for $0 \leq \alpha \leq 1$.

Let us recall the following known definitions of fractional calculus. For more details, see $[43,44]$.

Definition 2 The fractional integral of order $\alpha>0$ with the lower limit 0 for a function $f$ is defined as

$$
I^{\alpha} f(t)=\frac{1}{\Gamma(\alpha)} \int_{0}^{t} \frac{f(s)}{(t-s)^{1-\alpha}} d s, \quad t>0, \alpha>0
$$

provided the right-hand side is pointwise defined on $[0, \infty)$, where $\Gamma$ is the gamma function.

Definition 3 The Caputo derivative of order $\alpha>0$ with the lower limit 0 for a function $f$ can be written as

$$
{ }^{C} D^{\alpha} f(t)=\frac{1}{\Gamma(n-\alpha)} \int_{0}^{t} \frac{f^{(n)}(s)}{(t-s)^{\alpha+1-n}} d s=I^{n-\alpha} f^{(n)}(t), \quad t>0,0 \leq n-1<\alpha<n .
$$

The Caputo derivative of a constant is equal to zero. If $f$ is an abstract function with values in $X$ then the integrals which appear in Definitions 2 and 3 are taken in Bochner's sense.

According to Definitions 2 and 3, it is suitable to rewrite the problem (1) in the equivalent integral equation

$$
\begin{aligned}
x(t)= & x_{0}+\frac{1}{\Gamma(q)} \int_{0}^{t}(t-s)^{\alpha-1} \\
& \times\left[A x(s)+B u(s)+f(s, x(s))+\int_{0}^{s} K(s-r) g(r, x(r)) d r\right] d s, \quad t \in[0, T],
\end{aligned}
$$

provided that the integral in (2) exists. Applying the Laplace transform

$$
\begin{aligned}
& v(\lambda)=\int_{0}^{\infty} e^{-\lambda s} x(s) d s, \quad w(\lambda)=\int_{0}^{\infty} e^{-\lambda s} u(s) d s \quad \text { and } \\
& \omega(\lambda)=\int_{0}^{\infty} e^{-\lambda s}\left(f(s, x(s))+\int_{0}^{s} K(s-r) g(r, x(r)) d r\right) d s, \quad \lambda>0,
\end{aligned}
$$


to (2) and using the method similar to that used in [38] we get

$$
\begin{aligned}
x(t)= & \int_{0}^{\infty} \Psi_{\beta}(\theta) S\left(t^{\beta} \theta\right) x_{0} d \theta+\beta \int_{0}^{t} \int_{0}^{\infty} \theta(t-s)^{\alpha-1} \Psi_{\beta}(\theta) S\left((t-s)^{\beta} \theta\right) \\
& \times\left[B u(s)+\left(f(s, x(s))+\int_{0}^{s} K(s-r) g(r, x(r)) d r\right)\right] d \theta d s,
\end{aligned}
$$

where

$$
\begin{aligned}
& \Psi_{\beta}(\theta)=\frac{1}{\beta} \theta^{-1-\frac{1}{\beta}} \bar{w}_{q}\left(\theta^{-\frac{1}{\beta}}\right) \geq 0, \\
& \bar{w}_{\beta}(\theta)=\frac{1}{\pi} \sum_{n=1}^{\infty}(-1)^{n-1} \theta^{-\beta n-1} \frac{\Gamma(n \beta+1)}{n !} \sin (n \pi \beta), \quad \theta \in(0, \infty) .
\end{aligned}
$$

Here, $\Psi_{\beta}$ is a probability density function defined on $(0, \infty)$, that is $\Psi_{\beta}(\theta) \geq 0, \theta \in(0, \infty)$ and $\int_{0}^{\infty} \Psi_{\beta}(\theta) d \theta=1$.

For $x \in X$, we define two families $\left\{S_{\beta}(t): t \geq 0\right\}$ and $\left\{P_{\beta}(t): t \geq 0\right\}$ of operators by

$$
\begin{aligned}
& S_{\beta}(t)=\int_{0}^{\infty} \Psi_{\beta}(\theta) S\left(t^{\beta} \theta\right) d \theta, \\
& P_{\beta}(t)=\beta \int_{0}^{\infty} \theta \Psi_{\beta}(\theta) S\left(t^{\beta} \theta\right) d \theta,
\end{aligned}
$$

respectively.

The following lemma follows from the results given in [37-39].

Lemma 4 The operators $S_{\beta}$ and $P_{\beta}$ have the following properties.

(i) For any fixed $t \geq 0$, and any $x \in X_{\alpha}$, we have the operators $S_{\beta}(t)$ and $P_{\beta}(t)$ are linear and bounded operators, i.e. for any $x \in X$,

$$
\left\|S_{\beta}(t) x\right\|_{\alpha} \leq M\|x\|_{\alpha} \quad \text { and } \quad\left\|P_{\beta}(t) x\right\|_{\alpha} \leq \frac{M}{\Gamma(\beta)}\|x\|_{\alpha} .
$$

(ii) The operators $S_{\beta}(t)$ and $P_{\beta}(t)$ are strongly continuous for all $t \geq 0$.

(iii) $S_{\beta}(t)$ and $P_{\beta}(t)$ are norm continuous in $X$ for $t>0$.

(iv) $S_{\beta}(t)$ and $P_{\beta}(t)$ are compact operators in $X$ for $t>0$.

(v) For every $t>0$, the restriction of $S_{\beta}(t)$ to $X_{\alpha}$ and the restriction of $P_{\beta}(t)$ to $X_{\alpha}$ are norm continuous.

(vi) For every $t>0$, the restriction of $S_{\beta}(t)$ to $X_{\alpha}$ and the restriction of $P_{\beta}(t)$ to $X_{\alpha}$ are compact operators in $X_{\alpha}$.

(vii) For all $x \in X$ and $t \in(0, \infty)$,

$$
\left\|A^{\alpha} P_{\beta}(t) x\right\| \leq C_{\alpha} t^{-\alpha \beta}\|x\|, \quad \text { where } C_{\alpha}:=\frac{M_{\alpha} \beta \Gamma(2-\alpha)}{\Gamma(1+\beta(1-\alpha))}
$$

In this paper, we adopt the following definition of mild solution of equation (1). 
Definition 5 A function $x\left(\cdot ; x_{0}, u\right) \in C\left([0, T], X_{\alpha}\right)$ is said to be a mild solution of (1) if for any $u \in L_{2}([0, T], U)$ the integral equation

$$
\begin{aligned}
x(t)= & S_{\beta}(t)\left(x_{0}+h(x)\right)+\int_{0}^{t}(t-s)^{\beta-1} P_{\beta}(t-s) B u(s) d s \\
& +\int_{0}^{t}(t-s)^{\beta-1} P_{\beta}(t-s)\left[f(s, x(s))+\int_{0}^{s} K(s-r) g(r, x(r)) d r\right] d s,
\end{aligned}
$$

is satisfied.

It is clear that $L_{0}^{t}:=\int_{0}^{t}(t-s)^{\beta-1} P_{\beta}(t-s) B u(s) d s: L_{2}([0, T], U) \rightarrow C\left([0, T], X_{\alpha}\right)$ is bounded if $\frac{1}{2}<\beta \leq 1$. In what follows, we assume that $\frac{1}{2}<\beta \leq 1$.

\section{Approximate controllability}

In this section, we state and prove conditions for the approximate controllability of semilinear fractional control integro-differential systems. To do this, we first prove the existence of a fixed point of the operator $\Lambda_{\varepsilon}$ defined below using Krasnoselskii's fixed-point theorem. Secondly, in Theorem 11, we show that under the uniform boundedness of $f$ and $g$ the approximate controllability of fractional systems (1) is implied by the approximate controllability of the corresponding linear system (4).

Let $x\left(T ; x_{0}, u\right)$ be the state value of $(1)$ at terminal time $T$ corresponding to the control $u$ and the initial value $x_{0}$. Introduce the set $\Re\left(T, x_{0}\right)=\left\{x\left(T ; x_{0}, u\right): u \in L_{2}([0, T], U)\right\}$, which is called the reachable set of system (1) at terminal time $T$, its closure in $X_{\alpha}$ is denoted by $\overline{\Re\left(T, x_{0}\right)}$.

Definition 6 The system (1) is said to be approximately controllable on $[0, T]$ if $\overline{\Re\left(T, x_{0}\right)}=$ $X_{\alpha}$, that is, given an arbitrary $\varepsilon>0$ it is possible to steer from the point $x_{0}$ to within a distance $\varepsilon$ from all points in the state space $X_{\alpha}$ at time $T$.

Consider the following linear fractional differential system:

$$
\begin{aligned}
& D_{t}^{\beta} x(t)=A x(t)+B u(t), \quad t \in[0, T], \\
& x(0)=x_{0} .
\end{aligned}
$$

The approximate controllability for linear fractional system (4) is a natural generalization of approximate controllability of linear first order control system $[9,10,12]$. It is convenient at this point to introduce the controllability and resolvent operators associated with (4) as

$$
\begin{aligned}
& \Gamma_{0}^{T}=\int_{0}^{T}(T-s)^{\beta-1} P_{\beta}(T-s) B B^{*} P_{\beta}^{*}(T-s) d s: X \rightarrow X, \\
& R\left(\varepsilon, \Gamma_{0}^{T}\right)=\left(\varepsilon I+\Gamma_{0}^{T}\right)^{-1}: X \rightarrow X, \quad \varepsilon>0,
\end{aligned}
$$

respectively, where $B^{*}$ denotes the adjoint of $B$ and $P_{\beta}^{*}(t)$ is the adjoint of $P_{\beta}(t)$. It is straightforward that the operator $\Gamma_{0}^{T}$ is a linear bounded operator.

Theorem 7 [10] Let $Z$ be a separable reflexive Banach space and let $Z^{*}$ stands for its dual space. Assume that $\Gamma: Z^{*} \rightarrow Z$ is symmetric. Then the following two conditions are equivalent: 
1. $\Gamma: Z^{*} \rightarrow Z$ is positive, that is, $\left\langle z^{*}, \Gamma z^{*}\right\rangle>0$ for all nonzero $z^{*} \in Z^{*}$.

2. For all $h \in Z z_{\varepsilon}(h)=\varepsilon(\varepsilon I+\Gamma J)^{-1}(h)$ strongly converges to zero as $\varepsilon \rightarrow 0^{+}$. Here, $J$ is the duality mapping of $Z$ into $Z^{*}$.

Lemma 8 The linear fractional control system (4) is approximately controllable on $[0, T]$ if and only if $\varepsilon R\left(\varepsilon, \Gamma_{0}^{T}\right) \rightarrow 0$ as $\varepsilon \rightarrow 0^{+}$in the strong operator topology.

Proof The lemma is a straightforward consequence of Theorem 7. Indeed, the system (4) is approximately controllable on $[0, T]$ if and only if $\left\langle\Gamma_{0}^{T} x, x\right\rangle>0$ for all nonzero $x \in X$, see [7]. By Theorem 7, $\left\|\varepsilon\left(\varepsilon I+\Gamma_{0}^{T}\right)^{-1}(h)\right\| \rightarrow 0$ as $\varepsilon \rightarrow 0^{+}$for all $h \in X$.

Remark 9 Notice that positivity of $\Gamma_{0}^{T}$ is equivalent to $\left\langle\Gamma_{0}^{T} x, x\right\rangle=0 \Longrightarrow x=0$. In other words, since $\left\langle\Gamma_{0}^{T} x, x\right\rangle=\int_{0}^{T}(T-s)^{\beta-1}\left\|B^{*} P_{\beta}^{*}(T-s) x\right\|^{2} d s$, approximate controllability of the linear system (4) is equivalent to $B^{*} P_{\beta}^{*}(T-s) x=0,0 \leq s<T \Longrightarrow x=0$.

Before proving the main results, let us first introduce our basic assumptions.

( $\left.\mathrm{H}_{1}\right) f, g:[0, T] \times X_{\alpha} \times X_{\alpha} \rightarrow X$ are continuous and for each $r \in \mathbb{N}$, there exists a constant $\gamma \in[0, \beta(1-\alpha)]$ and functions $\varphi_{r} \in L^{1 / \gamma}\left([0, T] ; \mathbb{R}^{+}\right), \psi_{r} \in L^{\infty}\left([0, T] ; \mathbb{R}^{+}\right)$such that

$$
\begin{aligned}
& \sup \left\{\|f(t, x)\|:\|x\|_{\alpha} \leq r\right\} \leq \varphi_{r} \quad \text { and } \quad \lim _{r \rightarrow \infty} \frac{\left\|\varphi_{r}\right\|_{L^{1 / \gamma}}}{r}=\sigma_{1}<\infty, \\
& \sup \left\{\|g(t, x)\|:\|x\|_{\alpha} \leq r\right\} \leq \psi_{r} \quad \text { and } \quad \lim _{r \rightarrow \infty} \frac{\left\|\psi_{r}\right\|_{L^{\infty}}}{r}=\sigma_{2}<\infty .
\end{aligned}
$$

$\left(\mathrm{H}_{2}\right) h: C\left([0, T] ; X_{\alpha}\right) \rightarrow X_{\alpha}$ is a Lipschitz function with Lipschitz constant $L_{h}$.

$\left(\mathrm{H}_{c}\right)$ The linear system (4) is approximately controllable on $[0, T]$.

Using the hypothesis $\left(\mathrm{H}_{c}\right)$, for an arbitrary function $x \in C\left([0, T] ; X_{\alpha}\right)$, we choose the feedback control function as follows:

$$
\begin{aligned}
u_{\varepsilon}(t, x)= & B^{*} P_{\beta}^{*}(T-t)\left(\varepsilon I+\Gamma_{0}^{T}\right)^{-1}\left[S_{\beta}(T)\left(x_{0}+h(x)\right)\right. \\
& \left.-\int_{0}^{T}(T-s)^{\beta-1} P_{\beta}(T-s)\left[f(s, x(s))+\int_{0}^{s} K(s, r) g(r, x(r)) d r\right] d s\right] .
\end{aligned}
$$

Let $B_{r}=\left\{x \in C\left([0, T] ; X_{\alpha}\right):\|x\|_{\alpha} \leq r\right\}$, where $r$ is a positive constant. Then $B_{r}$ is clearly a bounded closed and convex subset in $C\left([0, T] ; X_{\alpha}\right)$. We will show that when using the above control the operator $\Lambda_{\varepsilon}: B_{k} \rightarrow B_{k}$ defined by

$$
\left(\Lambda_{\varepsilon} x\right)(t):=\left(\Phi_{\varepsilon} x\right)(t)+\left(\Pi_{\varepsilon} x\right)(t), \quad t \in[0, T],
$$

where

$$
\begin{aligned}
\left(\Phi_{\varepsilon} x\right)(t):= & S_{\beta}(t)\left(x_{0}+h(x)\right), \\
\left(\Pi_{\varepsilon} x\right)(t):= & \int_{0}^{t}(t-s)^{\beta-1} P_{\beta}(t-s)\left[f(s, x(s))+\int_{0}^{s} K(s, r) g(r, x(r)) d r\right] d s \\
& +\int_{0}^{t}(t-s)^{\beta-1} P_{\beta}(t-s) B u_{\varepsilon}(s, x) d s
\end{aligned}
$$

has a fixed point in $C\left([0, T] ; X_{\alpha}\right)$. 
Theorem 10 Let the assumptions $\left(\mathrm{H}_{1}\right)$ and $\left(\mathrm{H}_{2}\right)$ be satisfied. Then for $x_{0} \in X_{\alpha}$, the fractional Cauchy problem (1) with $u=u_{\varepsilon}(t, x)$ has at least one mild solution provided that

$$
L_{C}+\frac{C_{\alpha} T^{(1-\alpha) \beta}}{\varepsilon(1-\alpha) \beta} \frac{M}{\Gamma(\beta)} L_{B}^{2} L_{C}<1
$$

where

$$
\begin{aligned}
& L_{C}:=M L_{h}+C_{\alpha}\left(\frac{1-\gamma}{(1-\alpha) \beta-\gamma}\right)^{1-\gamma} T^{(1-\alpha) \beta-\gamma} \sigma_{1}+\frac{C_{\alpha} K T^{(1-\alpha) \beta}}{(1-\alpha) \beta} \sigma_{2}, \\
& L_{B}:=\|B\|, \quad K:=\max _{0 \leq t \leq b}|K(t)| .
\end{aligned}
$$

Proof It is easy to see that for any $\varepsilon>0$ the operator $\Lambda_{\varepsilon}$ maps $C\left([0, T] ; X_{\alpha}\right)$ into itself.

Let $x \in B_{r}$ and $0 \leq t \leq T$. Using assumption $\left(\mathrm{H}_{1}\right)$ yield the following estimations,

$$
\begin{aligned}
\left\|u_{\varepsilon}(s, x)\right\| \leq & \frac{1}{\varepsilon} \frac{M}{\Gamma(\beta)} L_{B}\left[M\left(\left\|x_{0}\right\|_{\alpha}+L_{h} r+\|h(0)\|_{\alpha}\right)\right. \\
& \left.+C_{\alpha}\left(\frac{1-\gamma}{(1-\alpha) \beta-\gamma}\right)^{1-\gamma} T^{(1-\alpha) \beta-\gamma}\left\|\varphi_{r}\right\|_{L^{1 / \gamma}}+\frac{C_{\alpha} K T^{(1-\alpha) \beta}}{(1-\alpha) \beta}\left\|\psi_{r}\right\|_{L^{\infty}}\right] \\
\leq & \frac{1}{\varepsilon} \frac{M}{\Gamma(\beta)} L_{B} L_{u}(r), \\
L_{u}(r):= & M\left(\left\|x_{0}\right\|_{\alpha}+L_{h} r+\|h(0)\|_{\alpha}\right)+C_{\alpha}\left(\frac{1-\gamma}{(1-\alpha) \beta-\gamma}\right)^{1-\gamma} T^{(1-\alpha) \beta-\gamma}\left\|\varphi_{r}\right\|_{L^{1 / \gamma}} \\
+ & \frac{C_{\alpha} K T^{(1-\alpha) \beta}}{(1-\alpha) \beta}\left\|\psi_{r}\right\|_{L^{\infty}},
\end{aligned}
$$

and

$$
\begin{aligned}
& \left\|\left(\Phi_{\varepsilon} y\right)(t)+\left(\Pi_{\varepsilon} x\right)(t)\right\|_{\alpha} \\
& \leq\left\|S_{\beta}(t)\left(x_{0}-h(y)\right)\right\|_{\alpha}+\int_{0}^{t}(t-s)^{\beta-1}\left\|A^{\alpha} P_{\beta}(t-s)\right\|_{L(X)}\left\|B u_{\varepsilon}(s, x)\right\| d s \\
& \quad+\int_{0}^{t}(t-s)^{\beta-1}\left\|A^{\alpha} P_{\beta}(t-s)\right\|_{L(X)}\left\|f(s, x(s))+\int_{0}^{s} K(s, r) g(r, x(r)) d r\right\| d s \\
& \leq M\left(\left\|x_{0}\right\|_{\alpha}+L_{h} r+\|h(0)\|_{\alpha}\right)+C_{\alpha} \int_{0}^{t}(t-s)^{\beta(1-\alpha)-1} \frac{1}{\varepsilon} L_{B}^{2} \frac{M}{\Gamma(\beta)} L_{u} d s \\
& \quad+C_{\alpha} \int_{0}^{t}(t-s)^{\beta(1-\alpha)-1}\left(\varphi_{r}(s)+K\left\|\psi_{r}\right\|_{L^{\infty}}\right) d s \\
& \leq M\left(\left\|x_{0}\right\|_{\alpha}+L_{h} r+\|h(0)\|_{\alpha}\right)+\frac{C_{\alpha} T^{(1-\alpha) \beta}}{\varepsilon(1-\alpha) \beta} \frac{M}{\Gamma(\beta)} L_{B}^{2} L_{u}(r) \\
& \quad+C_{\alpha}\left(\frac{1-\gamma}{(1-\alpha) \beta-\gamma}\right)^{1-\gamma} T^{(1-\alpha) \beta-\gamma}\left\|\varphi_{r}\right\|_{L^{1 / \gamma}} \\
& \quad+\frac{C_{\alpha} K T^{(1-\alpha) \beta}}{(1-\alpha) \beta}\left\|\psi_{r}\right\|_{L^{\infty}} .
\end{aligned}
$$


From (6) and the assumption $\left(\mathrm{H}_{2}\right)$, it follows that for any $\varepsilon>0$ there exists $r(\varepsilon)>0$ such that

$$
\begin{aligned}
& M\left(\left\|x_{0}\right\|_{\alpha}+L_{h} r(\varepsilon)+\|h(0)\|_{\alpha}\right)+\frac{C_{\alpha} T^{(1-\alpha) \beta}}{\varepsilon(1-\alpha) \beta} \frac{M}{\Gamma(\beta)} L_{B}^{2} L_{u}(r(\varepsilon)) \\
& \quad+C_{\alpha}\left(\frac{1-\gamma}{(1-\alpha) \beta-\gamma}\right)^{1-\gamma} T^{(1-\alpha) \beta-\gamma}\left\|\varphi_{r(\varepsilon)}\right\|_{L^{1 / \gamma}}+\frac{C_{\alpha} K T^{(1-\alpha) \beta}}{(1-\alpha) \beta}\left\|\psi_{r(\varepsilon)}\right\|_{L^{\infty}} \leq r(\varepsilon) .
\end{aligned}
$$

Therefore, from (7) and (8), it follows that for any $\varepsilon>0$ there exists $r(\varepsilon)>0$ such that $\Phi_{\varepsilon} y+$ $\Pi_{\varepsilon} x \in B_{r(\varepsilon)}$ for every $x, y \in B_{r(\varepsilon)}$. Therefore, for any $\varepsilon>0$ the fractional Cauchy problem (1) with the control (5) has a mild solution if and only if the operator $\Phi_{\varepsilon}+\Pi_{\varepsilon}$ has a fixed point in $B_{r(\varepsilon)}$.

In what follows, we will show that $\Phi_{\varepsilon}$ and $\Pi_{\varepsilon}$ satisfy the conditions of Krasnoselskii's fixed-point theorem. From $\left(\mathrm{H}_{2}\right)$ and (6), we infer that $\Phi_{\varepsilon}$ is a contraction. Next, we show that $\Pi_{\varepsilon}$ is completely continuous on $B_{r(\varepsilon)}$.

Step 1: We first prove that $\Pi_{\varepsilon}$ is continuous on $B_{r(\varepsilon)}$. Let $\left\{x_{n}\right\}_{n=1}^{\infty} \subset B_{r(\varepsilon)}$ be a sequence such that $x_{n} \rightarrow x$ as $n \rightarrow \infty$ in $C\left([0, T] ; X_{\alpha}\right)$. Therefore, it follows from the continuity of $f$, $g$ and $u_{\varepsilon}$ that for each $t \in[0, T]$,

$$
\begin{aligned}
& f\left(s, x_{n}(s)\right) \rightarrow f(s, x(s)), \\
& g\left(s, x_{n}(s)\right) \rightarrow g(s, x(s)), \\
& u_{\varepsilon}\left(s, x_{n}(s)\right) \rightarrow B u_{\varepsilon}(s, x(s)) \quad \text { as } n \rightarrow \infty .
\end{aligned}
$$

Also, by $\left(\mathrm{H}_{1}\right)$, we see that

$$
\begin{aligned}
\int_{0}^{t}(t & -s)^{\beta-1-\alpha \beta}\left(\left\|f\left(s, x_{n}(s)\right)-f(s, x(s))\right\|\right. \\
& \left.+\int_{0}^{s}|K(s-r)|\left\|g\left(r, x_{n}(r)\right)-g(r, x(r))\right\| d r\right) d s \\
& +\int_{0}^{t}(t-s)^{\beta-1-\alpha \beta}\left\|B u_{\varepsilon}\left(s, x_{n}(s)\right)-B u_{\varepsilon}(s, x(s))\right\| d s \\
\leq & 2 C_{\alpha}\left(\frac{1-\gamma}{(1-\alpha) \beta-\gamma}\right)^{1-\gamma} T^{(1-\alpha) \beta-\gamma}\left\|\varphi_{k}\right\|_{L^{1 / \gamma}}+\frac{2 C_{\alpha} K T^{(1-\alpha) \beta}}{(1-\alpha) \beta}\left\|\psi_{k}\right\|_{L^{\infty}} \\
& +C_{\alpha} \frac{1}{\varepsilon} \int_{0}^{t}(t-s)^{\beta(1-\alpha)-1} \frac{M}{\Gamma(\beta)} L_{B}^{2} L_{u} d s .
\end{aligned}
$$

Since

$$
\begin{aligned}
& \left\|\left(\Pi_{\varepsilon} x_{n}\right)(t)-\left(\Pi_{\varepsilon} x\right)(t)\right\|_{\alpha} \\
& \leq C_{\alpha} \int_{0}^{t}(t-s)^{\beta-1-\alpha \beta} \\
& \quad \times\left(\left\|f\left(s, x_{n}(s)\right)-f(s, x(s))\right\|+\int_{0}^{s}|K(s-r)|\left\|g\left(r, x_{n}(r)\right)-g(r, x(r))\right\| d r\right) d s,
\end{aligned}
$$


using the Lebesgue dominated convergence theorem that for all $t \in[0, T]$, we conclude

$$
\left\|\left(\Pi_{\varepsilon} x_{n}\right)(t)-\left(\Pi_{\varepsilon} x\right)(t)\right\|_{\alpha} \rightarrow 0, \quad \text { as } n \rightarrow \infty,
$$

implying that $\left\|\Pi_{\varepsilon} x_{n}-\Pi_{\varepsilon} x\right\|_{\alpha} \rightarrow 0$ as $n \rightarrow \infty$. This proves that $\Pi_{\varepsilon}$ is continuous on $B_{r(\varepsilon)}$.

Step $2 . \Pi_{\varepsilon}$ is compact on $B_{r(\varepsilon)}$.

For the sake of brevity, we write

$$
N(x(s)):=f(s, x(s))+\int_{0}^{s} K(s, r) g(r, x(r)) d r+B u_{\varepsilon}(s, x) .
$$

Let $t \in[0, T]$ be fixed and $\delta, \eta>0$ be small enough. For $x \in B_{r(\varepsilon)}$, we define the map

$$
\begin{aligned}
\left(\Pi_{\varepsilon}^{\delta \eta} x\right)(t) & =\int_{0}^{\delta} \int_{\eta}^{\infty} \beta r(t-s)^{\beta-1} \Psi_{\beta}(r) S\left((t-s)^{\beta} r\right) N(x(s)) d r d s \\
& =S\left(\delta^{\beta} \eta\right) \int_{0}^{\delta} \int_{\eta}^{\infty} \beta r(t-s)^{\beta-1} \Psi_{\beta}(r) S\left((t-s)^{\beta} r-\delta^{\beta} \eta\right) N(x(s)) d r d s .
\end{aligned}
$$

Therefore, from Lemma 4, we see that for each $t \in(0, T]$, the set $\left\{\left(\Pi_{\varepsilon}^{\delta \eta} x\right)(t): x \in B_{r(\varepsilon)}\right\}$ is relatively compact in $X_{\alpha}$. Since

$$
\begin{aligned}
\|( & \left.\Pi_{\varepsilon} x\right)(t)-\left(\Pi_{\varepsilon}^{\delta \eta} x\right)(t) \|_{\alpha} \\
\leq & \left\|\int_{0}^{t} \int_{0}^{\eta} \beta r(t-s)^{\beta-1} \Psi_{\beta}(r) S\left((t-s)^{\beta} r\right) N(x(s)) d r d s\right\|_{\alpha} \\
& +\left\|\int_{t-\delta}^{t} \int_{\eta}^{\infty} \beta r(t-s)^{\beta-1} \Psi_{\beta}(r) S\left((t-s)^{\beta} r\right) N(x(s)) d r d s\right\|_{\alpha} \\
\leq & \beta M_{\alpha}\left[\int_{0}^{t}(t-s)^{\beta(1-\alpha)-1}\left(\varphi_{r(\varepsilon)}(s)+K\left\|\psi_{r(\varepsilon)}\right\|_{L^{\infty}}+\frac{1}{\varepsilon} \frac{M}{\Gamma(\beta)} L_{B}^{2} L_{u}\right) d s \int_{0}^{\eta} \tau^{1-\alpha} \Psi_{\beta}(\tau) d \tau\right. \\
& \left.+\int_{t-\delta}^{t}(t-s)^{\beta(1-\alpha)-1}\left(\varphi_{r(\varepsilon)}(s)+K\left\|\psi_{r(\varepsilon)}\right\|_{L^{\infty}}+\frac{1}{\varepsilon} \frac{M}{\Gamma(\beta)} L_{B}^{2} L_{u}\right) d s \int_{\eta}^{\infty} \tau^{1-\alpha} \Psi_{\beta}(\tau) d \tau\right] \\
\leq & \beta M_{\alpha}\left[\left(\frac{1-\gamma}{(1-\alpha) \beta-\gamma}\right)^{1-\gamma} T^{(1-\alpha) \beta-\gamma}\left\|\varphi_{r(\varepsilon)}\right\|_{L^{1 / \gamma}}+\frac{K T^{(1-\alpha) \beta}}{(1-\alpha) \beta}\left\|\psi_{r(\varepsilon)}\right\|_{L^{\infty}}\right. \\
& \left.+\frac{T^{(1-\alpha) \beta}}{(1-\alpha) \beta} \frac{1}{\varepsilon} \frac{M}{\Gamma(\beta)} L_{B}^{2} L_{u}\right] \int_{0}^{\eta} \tau^{1-\alpha} \Psi_{\beta}(\tau) d \tau \\
& +\frac{\beta M_{\alpha} \Gamma(2-\alpha)}{\Gamma(1+\beta(1-\alpha))}\left[\left(\frac{1-\gamma}{(1-\alpha) \beta-\gamma}\right)^{1-\gamma} \eta^{(1-\alpha) \beta-\gamma}\left\|\varphi_{r(\varepsilon)}\right\|_{L^{1 / \gamma}}+\frac{K \eta^{(1-\alpha) \beta}}{(1-\alpha) \beta}\left\|\psi_{r(\varepsilon)}\right\|_{L^{\infty}}\right. \\
& \left.+\frac{\eta^{(1-\alpha) \beta}}{(1-\alpha) \beta} \frac{1}{\varepsilon} \frac{M}{\Gamma(\beta)} L_{B}^{2} L_{u}\right]
\end{aligned}
$$

approaches to zero as $\eta \rightarrow 0^{+}$, using the total boundedness, we conclude that for each $t \in[0, T]$, the set $\left\{\left(\Pi_{\varepsilon}^{\delta \eta} x\right)(t): x \in B_{r(\varepsilon)}\right\}$ is relatively compact in $X_{\alpha}$.

On the other hand, for $0<t_{1}<t_{2} \leq T$ and $\delta>0$ small enough, we have

$$
\left\|\left(\Pi_{\varepsilon} x\right)\left(t_{1}\right)-\left(\Pi_{\varepsilon} x\right)\left(t_{2}\right)\right\|_{\alpha} \leq I_{1}+I_{2}+I_{3}+I_{4},
$$


where

$$
\begin{aligned}
& I_{1}:=\int_{t_{1}}^{t_{2}}\left(t_{2}-s\right)^{\beta-1-\alpha \beta}\|N(x(s))\| d s, \\
& I_{2}:=\int_{0}^{t_{1}-\delta}\left(t_{1}-s\right)^{\beta-1}\left\|A^{\alpha} P_{\beta}\left(t_{2}-s\right)-A^{\alpha} P_{\beta}\left(t_{1}-s\right)\right\|_{L(X)}\|N(x(s))\| d s, \\
& I_{3}:=\int_{t_{1}-\delta}^{t_{1}}\left(t_{1}-s\right)^{\beta-1}\left(\left(t_{2}-s\right)^{-\alpha \beta}+\left(t_{1}-s\right)^{-\alpha \beta}\right)\|N(x(s))\| d s, \\
& I_{4}:=\int_{0}^{t_{1}}\left(t_{2}-s\right)^{-\alpha \beta}\left|\left(t_{2}-s\right)^{\beta-1}-\left(t_{1}-s\right)^{\beta-1}\right|\|N(x(s))\| d s .
\end{aligned}
$$

Therefore, it follows from $\left(\mathrm{H}_{1}\right)$ and Lemma 4 that

$$
\begin{aligned}
I_{1} \leq & C_{\alpha} \int_{t_{1}}^{t_{2}}\left(t_{2}-s\right)^{\beta-1-\alpha \beta}\left(\left(\varphi_{r(\varepsilon)}(s)+K\left\|\psi_{r(\varepsilon)}\right\|_{L^{\infty}}\right)+\frac{1}{\varepsilon} L_{B}^{2} \frac{M}{\Gamma(\beta)} L_{u}(r(\varepsilon))\right) d s \\
\leq & C_{\alpha}\left(\left(\frac{1-\gamma}{(1-\alpha) \beta-\gamma}\right)^{1-\gamma}\left(t_{2}-t_{1}\right)^{(1-\alpha) \beta-\gamma}\left\|\varphi_{r(\varepsilon)}\right\|_{L^{1 / \gamma}}\right. \\
& +\frac{C_{\alpha} K\left(t_{2}-t_{1}\right)^{(1-\alpha) \beta}}{(1-\alpha) \beta}\left\|\psi_{r(\varepsilon)}\right\|_{L^{\infty}} \\
& \left.+\frac{C_{\alpha}^{(1-\alpha) \beta}\left(t_{2}-t_{1}\right)}{\varepsilon(1-\alpha) \beta} \frac{M}{\Gamma(\beta)} L_{B}^{2} L_{u}(r(\varepsilon))\right), \\
I_{2} \leq & \sup _{0 \leq s \leq t_{1}-\delta}\left\|A^{\alpha} P_{\beta}\left(t_{2}-s\right)-A^{\alpha} P_{\beta}\left(t_{1}-s\right)\right\|_{L(X)} \\
& \times \int_{0}^{t_{1}-\delta}\left(t_{2}-s\right)^{\beta-1-\alpha \beta}\left(\left(\varphi_{r(\varepsilon)}(s)+K\left\|\psi_{r(\varepsilon)}\right\|_{L^{\infty}}\right)+\frac{1}{\varepsilon} \frac{M}{\Gamma(\beta)} L_{B}^{2} L_{u}(r(\varepsilon))\right) d s \\
\leq & \sup _{0 \leq s \leq t_{1}-\delta}\left\|A^{\alpha} P_{\beta}\left(t_{2}-s\right)-A^{\alpha} P_{\beta}\left(t_{1}-s\right)\right\|_{L(X)} \\
& \times\left[\left(\frac{1-\gamma}{\beta-\gamma}\right)^{1-\gamma}\left\|\varphi_{r(\varepsilon)}\right\|_{L^{1 / \gamma}}\left(t_{1}^{\frac{\beta-\gamma}{1-\gamma}}-\delta^{\frac{\beta-\gamma}{1-\gamma}}\right)^{1-\gamma}+\frac{K\left\|\psi_{r(\varepsilon)}\right\|_{L^{\infty}}}{\beta}\left(t_{1}^{\beta}-\delta^{\beta}\right)\right. \\
& \left.+\frac{1}{\varepsilon \beta} \frac{M}{\Gamma(\beta)} L_{B}^{2} L_{u}(r(\varepsilon))\left(t_{1}^{\beta}-\delta^{\beta}\right)\right], \\
I_{3} \leq & 2 C_{\alpha} \int_{t_{1}-\delta}^{t_{1}}\left(t_{1}-s\right)^{\beta-1-\alpha \beta}\left(\left(\varphi_{k}(s)+K\left\|\psi_{k}\right\|_{L^{\infty}}\right)+\frac{1}{\varepsilon} \frac{M}{\Gamma(\beta)} L_{B}^{2} L_{u}\right) d s \\
\leq & 2 C_{\alpha}\left(\left(\frac{1-\gamma}{(1-\alpha) \beta-\gamma}\right)^{1-\gamma} \delta^{(1-\alpha) \beta-\gamma}\left\|\varphi_{k}\right\|_{L^{1 / \gamma}}+\frac{C_{\alpha} K\left\|\psi_{k}\right\|_{L^{\infty}}}{(1-\alpha) \beta} \delta^{(1-\alpha) \beta}\right. \\
& \left.+\frac{C_{\alpha} L_{B}^{2} L_{u}}{\varepsilon(1-\alpha) \beta} \frac{M}{\Gamma(\beta)} \delta^{(1-\alpha) \beta}\right), \\
& {\left[\left(\frac{1-\alpha}{1-\alpha}\right)\right.}
\end{aligned}
$$

and

$$
\begin{aligned}
I_{4} \leq & C_{\alpha} \int_{0}^{t_{1}}\left|\left(t_{1}-s\right)^{\beta-1-\alpha \beta}-\left(t_{2}-s\right)^{\beta-1-\alpha \beta}\right| \\
& \times\left(\left(\varphi_{r(\varepsilon)}(s)+K\left\|\psi_{r(\varepsilon)}\right\|_{L^{\infty}}\right)+\frac{1}{\varepsilon} \frac{M}{\Gamma(\beta)} L_{B}^{2} L_{u}(r(\varepsilon))\right) d s
\end{aligned}
$$




$$
\begin{aligned}
\leq & C_{\alpha}\left(\frac{1-\gamma}{(1-\alpha) \beta-\gamma}\right)^{1-\gamma}\left\|\varphi_{r(\varepsilon)}\right\|_{L^{1 / \gamma}}\left[t_{1}^{(1-\alpha) \beta-\gamma}-\left(t_{2}^{\frac{(1-\alpha) \beta-\gamma}{1-\gamma}}-\left(t_{2}-t_{1}\right)^{\frac{(1-\alpha) \beta-\gamma}{1-\gamma}}\right)^{1-\gamma}\right] \\
& +C_{\alpha} \frac{2 K}{(1-\alpha) \beta}\left\|\psi_{r(\varepsilon)}\right\|_{L^{\infty}}\left[t_{1}^{(1-\alpha) \beta}-t_{2}^{(1-\alpha) \beta}-\left(t_{2}-t_{1}\right)^{(1-\alpha) \beta}\right] \\
& +C_{\alpha} \frac{2 L_{B}^{2} L_{u}(r(\varepsilon))}{(1-\alpha) \beta} \frac{M}{\Gamma(\beta)}\left\|\psi_{r(\varepsilon)}\right\|_{L^{\infty}}\left[t_{1}^{(1-\alpha) \beta}-t_{2}^{(1-\alpha) \beta}-\left(t_{2}-t_{1}\right)^{(1-\alpha) \beta}\right],
\end{aligned}
$$

from which it is easy to see that all $I_{i}, i=1,2,3,4$, tend to zero independent of $x \in B_{k}$ as $t_{2}-t_{1} \rightarrow 0$ and $\delta \rightarrow 0$. Thus, we can conclude that

$$
\left\|\left(\Pi_{\varepsilon} x\right)\left(t_{1}\right)-\left(\Pi_{\varepsilon} x\right)\left(t_{2}\right)\right\|_{\alpha} \rightarrow 0 \quad \text { as } t_{2}-t_{1} \rightarrow 0,
$$

and the limit is independent of $x \in B_{r(\varepsilon)}$. The case $t_{1}=0$ is trivial. Consequently, the set $\left\{\left(\Pi_{\varepsilon} x\right)(t): t \in[0, T], x \in B_{r(\varepsilon)}\right\}$ is equicontinuous. Now applying the Arzela-Ascoli theorem, it results that $\Pi_{\varepsilon}$ is compact on $B_{r(\varepsilon)}$.

Therefore, applying Krasnoselskii's fixed-point theorem, we conclude that $\Lambda_{\varepsilon}$ has a fixed point, which gives rise to a mild solution of Cauchy problem (1) with control given in (5). This completes the proof.

Theorem 11 Let the assumptions $\left(\mathrm{H}_{1}\right),\left(\mathrm{H}_{2}\right)$ and $\left(\mathrm{H}_{c}\right)$ be satisfied. Moreover, assume the functions $f, g:[0, T] \times X_{\alpha} \times X_{\alpha} \rightarrow X$ and $h: C\left([0, T] ; X_{\alpha}\right) \rightarrow X_{\alpha}$ are bounded and $M L_{h}<1$. Then the semilinear fractional system (3) is approximately controllable on $[0, T]$.

Proof It is clear that all assumptions of Theorem 10 are satisfied with $\sigma_{1}=\sigma_{2}=0$. Let $x_{\varepsilon}$ be a fixed point of $F_{\varepsilon}$ in $B_{r}$. Any fixed point of $F_{\varepsilon}$ is a mild solution of (3) under the control

$$
\begin{aligned}
u_{\varepsilon}\left(t, x_{\varepsilon}\right)= & B^{*} P_{\beta}^{*}(T-t) R\left(\varepsilon, \Gamma_{0}^{T}\right)\left(h-S_{\beta}(T)\left(x_{0}+h\left(x_{\varepsilon}\right)\right)\right. \\
& \left.-\int_{0}^{T}(T-s)^{\beta-1} P_{\beta}(T-s)\left[f\left(s, x_{\varepsilon}(s)\right)+\int_{0}^{s} K(s-\tau) g\left(\tau, x_{\varepsilon}(\tau)\right) d \tau\right] d s\right)
\end{aligned}
$$

and satisfies the equality

$$
x_{\varepsilon}(T)=h-\varepsilon R\left(\varepsilon, \Gamma_{0}^{T}\right) p\left(x_{\varepsilon}\right)
$$

where

$$
\begin{aligned}
p\left(x_{\varepsilon}\right)= & \left(h-S_{\beta}(T)\left(x_{0}+h\left(x_{\varepsilon}\right)\right)\right. \\
& \left.-\int_{0}^{T}(T-s)^{\beta-1} P_{\beta}(T-s)\left[f\left(s, x_{\varepsilon}(s)\right)+\int_{0}^{s} K(s-\tau) g\left(\tau, x_{\varepsilon}(\tau)\right) d \tau\right] d s\right) .
\end{aligned}
$$

Moreover, by the boundedness of the functions $f$ and $g$ and Dunford-Pettis theorem, we have that the sequences $\left\{f\left(s, x_{\varepsilon}(s)\right)\right\}$ and $\left\{g\left(s, x_{\varepsilon}(s)\right)\right\}$ are weakly compact in $L^{2}([0, T] ; X)$, so there are subsequences still denoted by $\left\{f\left(s, x_{\varepsilon}(s)\right)\right\}$ and $\left\{g\left(s, x_{\varepsilon}(s)\right)\right\}$, that weakly converge to, say, $f$ and $g$ in $L^{2}([0, T] ; X)$. On the other hand, there exists $\tilde{h} \in X_{\alpha}$ such that $h\left(x_{\varepsilon}\right)$ converges to $\widetilde{h}$ weakly in $X_{\alpha}$. Denote

$$
\left.w=h-S_{\beta}\left(x_{0}+\widetilde{h}\right)\right)-\int_{0}^{T}(T-s)^{\beta-1} P_{\beta}(T-s)\left[f(s)+\int_{0}^{s} K(s-\tau) g(\tau) d \tau\right] d s .
$$


It follows that

$$
\begin{aligned}
\left\|p\left(x_{\varepsilon}\right)-w\right\|_{\alpha} \leq & \left\|S_{\beta}(T) h\left(x_{\varepsilon}\right)-S_{\beta}(T) \widetilde{h}\right\|_{\alpha} \\
& +\left\|\int_{0}^{T}(T-s)^{\beta-1} P_{\beta}(T-s)\left(f\left(s, x_{\varepsilon}(s)\right)-f(s)\right) d s\right\|_{\alpha} \\
& +\| \int_{0}^{T}(T-s)^{\beta-1} P_{\beta}(T-s) \int_{0}^{s} K(s-\tau)\left(g\left(\tau, x_{\varepsilon}(\tau)-g(\tau)\right) d \tau d s \|_{\alpha} \rightarrow 0\right.
\end{aligned}
$$

as $\varepsilon \rightarrow 0^{+}$because of compactness of the operator

$$
l(\cdot) \rightarrow \int_{0}^{\cdot}(\cdot-s)^{\beta-1} P_{\beta}(\cdot-s) l(s) d s: L_{2}([0, T], X) \rightarrow C\left([0, T], X_{\alpha}\right) .
$$

Then from (9), we obtain

$$
\begin{aligned}
\left\|x_{\varepsilon}(T)-h\right\|_{\alpha} & \leq\left\|\varepsilon R\left(\varepsilon, \Gamma_{0}^{T}\right)(w)\right\|_{\alpha}+\left\|\varepsilon R\left(\varepsilon, \Gamma_{0}^{T}\right)\right\|\left\|p\left(x_{\varepsilon}\right)-w\right\|_{\alpha} \\
& \leq\left\|\varepsilon R\left(\varepsilon, \Gamma_{0}^{T}\right)(w)\right\|_{\alpha}+\left\|p\left(x_{\varepsilon}\right)-w\right\|_{\alpha} \rightarrow 0
\end{aligned}
$$

as $\varepsilon \rightarrow 0^{+}$. This proves the approximate controllability of (1).

\section{Applications}

Example 1 As an application to Theorem 11, we study the following simple example. Consider a control system governed by the fractional partial differential equation of the form

$$
\left\{\begin{array}{l}
{ }^{c} \partial_{t}^{\frac{3}{4}} x(t, z)=\partial_{z}^{2} x(t, z)+u(t, z)+F(t, z, x(t, z)) \\
\quad+\int_{0}^{t} K(t, s) G(s, z, x(s, z)) d s, \quad t \in[0, T], z \in[0, \pi], \\
x(t, 0)=x(t, \pi)=0, \\
x(0, z)=x_{0}(z)+\sum_{k=1}^{p} \int_{0}^{\pi} k(z, r) \cos \left(x\left(t_{k}, r\right)\right) d r,
\end{array}\right.
$$

where $f, g:[0, T] \times[0, \pi] \times R \rightarrow R, k:[0, \pi] \times[0, \pi] \rightarrow R, 0<t_{1}<\cdots<t_{p}<T$.

Let us take $X=U=L^{2}[0, \pi]$ and define the operator $A$ by $A w=-w^{\prime \prime}$ with the domain $D(A)=\left\{w(\cdot) \in L^{2}[0, \pi], w, w^{\prime}\right.$ are absolutely continuous, $\left.w^{\prime \prime} \in L^{2}[0, \pi], w(0)=w(\pi)=0\right\}$. Then

$$
A w=\sum_{n=1}^{\infty} n^{2}\left\langle w, e_{n}\right\rangle e_{n}, \quad w \in D(A)
$$

where $e_{n}(z)=\sqrt{\frac{2}{\pi}} \sin n z, 0 \leq z \leq \pi, n=1,2, \ldots$. Clearly $-A$ generates a compact analytic semigroup $S(t), t>0$ in $X$ and it is given by

$$
S(t) w=\sum_{n=1}^{\infty} e^{-n^{2} t}\left\langle w, e_{n}\right\rangle e_{n}, \quad w \in X
$$

Clearly, the assumption $\left(\mathrm{H}_{1}\right)$ is satisfied. On the other hand, it can be easily seen that the deterministic linear system corresponding to (11) is approximately controllable on $[0, T]$; see [12]. 
The operator $A^{\frac{1}{2}}$ is given by

$$
A^{\frac{1}{2}} w=\sum_{n=1}^{\infty} n\left\langle w, e_{n}\right\rangle e_{n}, \quad w \in D\left(A^{\frac{1}{2}}\right),
$$

where $D\left(A^{\frac{1}{2}}\right)=\left\{w \in X: \sum_{n=1}^{\infty} n\left\langle w, e_{n}\right\rangle e_{n} \in X\right\}$ and $\left\|A^{-\frac{1}{2}}\right\|=1$.

Let $X_{\frac{1}{2}}:=\left(D\left(A^{\frac{1}{2}}\right),\|\cdot\|_{1 / 2}\right)$, where $\|x\|_{1 / 2}:=\left\|A^{\frac{1}{2}} x\right\|_{X}$ for $x \in D\left(A^{\frac{1}{2}}\right)$. Assume that $F, G$ :

$[0, T] \times[0, \pi] \times R \rightarrow R$ satisfies the following conditions:

1. The functions $F(\cdot, \cdot, \cdot), G(\cdot, \cdot, \cdot)$ are continuous and uniformly bounded.

2. $F(0, \cdot, \cdot)=F(\pi, \cdot, \cdot)=G(0, \cdot, \cdot)=G(\pi, \cdot, \cdot)=0$.

3. $k:[0, \pi] \times[0, \pi] \rightarrow R$ is continuously differentiable, $k(0, \cdot)=k(\pi, \cdot)=0$ and

$$
\int_{0}^{\pi} \int_{0}^{\pi}\left|\frac{\partial^{2}}{\partial \xi^{2}} k(\xi, y)\right|^{2} d y d \xi<\infty
$$

Denote by $E_{\beta, \zeta}$, the Mittag-Leffler special function defined by

$$
E_{\beta, \zeta}=\sum_{k=0}^{\infty} \frac{t^{k}}{\Gamma(\zeta k+\beta)}, \quad \zeta, \beta>0, t \in R .
$$

Therefore,

$$
\begin{array}{ll}
S_{\beta}(t) x= & \sum_{k=0}^{\infty} E_{\beta, 1}\left(-n^{2} t^{\beta}\right)\left\langle x, e_{n}\right\rangle e_{n}, \quad\left\|S_{\beta}(t)\right\|_{L(X)} \leq 1, \\
P_{\beta}(t) x=\sum_{k=0}^{\infty} E_{\beta, \beta}\left(-n^{2} t^{\beta}\right)\left\langle x, e_{n}\right\rangle e_{n}, \quad\left\|P_{\beta}(t)\right\|_{L(X)} \leq \frac{1}{\Gamma(\beta)}, \quad x \in X, t \geq 0 .
\end{array}
$$

Define

$$
\begin{aligned}
& f(t, x(t))(z)=F(t, z, x(t, z)), \\
& g(t, x(t))(z)=G(s, z, x(s, z)), \\
& h(x)(z)=\sum_{k=1}^{p} \int_{0}^{\pi} k(z, y) \cos \left(x\left(t_{k}, y\right)\right) d y .
\end{aligned}
$$

Then, for each $x, y \in C\left([0, T], X_{1 / 2}\right)$ we have

$$
\begin{aligned}
\|h(x)\|_{1 / 2}^{2} & =\left\|A^{1 / 2} h(x)(\cdot)\right\|_{L^{2}[0, \pi]}^{2}=\sum_{n=1}^{\infty} n^{2}\left\|e_{n}\right\|_{L^{2}[0, \pi]}^{2}\left|\left\langle h(x)(\cdot), e_{n}\right)\right|^{2} \\
& =\frac{2}{\pi} \sum_{n=1}^{\infty} n^{2}\left|\int_{0}^{\pi} h(x)(\xi) \sin (n \xi) d \xi\right|^{2}=\sum_{n=1}^{\infty} \frac{1}{n^{2}}\left|\int_{0}^{\pi} \frac{\partial^{2}}{\partial \xi^{2}} h(x)(\xi) e_{n}(\xi) d \xi\right|^{2} \\
& \leq \frac{\pi^{2}}{6}\left\|\frac{\partial^{2}}{\partial \xi^{2}} h(x)(\xi)\right\|_{L^{2}[0, \pi]}^{2}=\frac{\pi^{2}}{6}\left\|\frac{\partial^{2}}{\partial \xi^{2}} \sum_{k=0}^{p} \int_{0}^{\pi} k(\xi, y) \cos \left(x\left(t_{k}, y\right)\right) d y\right\|_{L^{2}[0, \pi]}^{2}
\end{aligned}
$$




$$
\begin{aligned}
& =\frac{\pi^{2}}{6} \int_{0}^{\pi}\left|\sum_{k=1}^{p} \int_{0}^{\pi} \frac{\partial^{2}}{\partial \xi^{2}} k(\xi, y) \cos \left(x\left(t_{k}, y\right)\right) d y\right|^{2} d \xi \\
& \leq \frac{p \pi^{3}}{6} \int_{0}^{\pi} \int_{0}^{\pi}\left|\frac{\partial^{2}}{\partial \xi^{2}} k(\xi, y)\right|^{2} d y d \xi=\frac{p \pi^{3}}{6}\left\|\frac{\partial^{2}}{\partial \xi^{2}} k(\xi, y)\right\|_{L^{2}[0, \pi] \times[0, \pi]}^{2}
\end{aligned}
$$

and

$$
\begin{aligned}
\|h(x)-h(y)\|_{1 / 2}^{2} & =\left\|A^{1 / 2} h(x)(\cdot)-A^{1 / 2} h(y)(\cdot)\right\|_{L^{2}[0, \pi]}^{2} \\
& \leq \frac{\pi^{2}}{6}\left\|\frac{\partial^{2}}{\partial \xi^{2}} \sum_{k=0}^{p} \int_{0}^{\pi} k(\xi, r)\left[\cos \left(x\left(t_{k}, r\right)\right)-\cos \left(y\left(t_{k}, r\right)\right)\right] d r\right\|_{L^{2}[0, \pi]}^{2} \\
& =\frac{\pi^{2}}{6} \int_{0}^{\pi}\left|\sum_{k=0}^{p} \int_{0}^{\pi} \frac{\partial^{2}}{\partial \xi^{2}} k(\xi, r)\left[\cos \left(x\left(t_{k}, r\right)\right)-\cos \left(y\left(t_{k}, r\right)\right)\right] d r\right|^{2} d \xi \\
& \leq \frac{p \pi^{2}}{6} \int_{0}^{\pi} \int_{0}^{\pi}\left|\frac{\partial^{2}}{\partial \xi^{2}} k(\xi, r)\right|^{2} d r d \xi \sup _{0 \leq t \leq \pi} \int_{0}^{\pi}|x(t, r)-y(t, r)|^{2} d r .
\end{aligned}
$$

It follows that $h: C\left([0, T] ; X_{1 / 2}\right) \rightarrow X_{1 / 2}$ is bounded and Lipschitz continuous. On the other hand, it is not difficult to verify that $f, g:[0, T] \times X_{1 / 2} \rightarrow X$ are continuous.

Next, we show that the linear system corresponding to (11) is approximately controllable on $[0, T]$. It is clear that $P_{\beta}(t): X_{\frac{1}{2}} \rightarrow X_{\frac{1}{2}}$ is defined as follows:

$$
\begin{aligned}
& P_{\beta}(t)=\beta \int_{0}^{\infty} \theta \Psi_{\beta}(\theta) S\left(t^{\beta} \theta\right) d \theta, \\
& B^{*} P_{\beta}^{*}(T-t) x \\
& \quad=\beta \sum_{n=1}^{\infty} n \int_{0}^{\infty} \theta \Psi_{\beta}(\theta) E_{\beta, \beta}\left(-n^{2}(T-t)^{\beta} \theta\right) d \theta\left\langle x, e_{n}\right\rangle e_{n}, \quad x \in X_{\frac{1}{2}}, 0 \leq t<T .
\end{aligned}
$$

By Remark 9, the linear system corresponding to (11) is approximately controllable on $[0, T]$ if and only if $B^{*} P_{\beta}^{*}(T-t) x=0,0 \leq t<T$ implies that $x=0$. This follows from the representation of $B^{*} P_{\beta}^{*}(T-t) x$.

Now, we note that the problem (11) can be reformulated as the abstract problem. Thus, by Theorem 11, the system (11) is approximately controllable on $[0, T]$, provided that

$$
M L_{h}=\frac{p \pi^{2}}{6} \int_{0}^{\pi} \int_{0}^{\pi}\left|\frac{\partial^{2}}{\partial \xi^{2}} k(\xi, r)\right|^{2} d r d \xi<1 .
$$

\section{Competing interests}

The authors declare that they have no competing interests.

\section{Authors' contributions}

All authors contributed equally and significantly in writing this paper. All authors read and approved the final manuscript.

\section{Acknowledgements}

Dedicated to Professor Hari M Srivastava.

The authors would like to thank the reviewers for their valuable comments and helpful suggestions that improved the note's quality.

Received: 12 December 2012 Accepted: 22 March 2013 Published: 8 May 2013 


\section{References}

1. Debbouche, A, Baleanu, D: Controllability of fractional evolution nonlocal impulsive quasilinear delay integro-differential systems. Comput. Math. Appl. 62(3), 1442-1450 (2011)

2. Agarwal, RP, Benchohra, M, Slimani, BA: Existence results for differential equations with fractional order and impulses. Mem. Differ. Equ. Math. Phys. 44, 1-21 (2008)

3. Abada, N, Benchohra, M, Hammouche, H: Existence and controllability results for nondensely defined impulsive semilinear functional differential inclusions. J. Differ. Equ. 246, 3834-3863 (2009)

4. Ahmad, B, Nieto, JJ: Existence results for a coupled system of nonlinear fractional differential equations with three-point boundary conditions. Comput. Math. Appl. 58, 1838-1843 (2009)

5. Bashirov, AE, Mahmudov, NI: On concepts of controllability for deterministic and stochastic systems. SIAM J. Control Optim. 37, 1808-1821 (1999)

6. Cannon, J: The One-Dimensional Heat Equation. Encyclopedia of Mathematics and Its Applications, vol. 23 Addison-Wesley, Menlo Park (1984)

7. Curtain, RF, Zwart, HJ: An Introduction to Infinite Dimensional Linear Systems Theory. Springer, New York (1995)

8. Dauer, JP, Mahmudov, NI: Approximate controllability of semilinear functional equations in Hilbert spaces. J. Math. Anal. Appl. 273, 310-327 (2002)

9. Mahmudov, NI, Denker, A: On controllability of linear stochastic systems. Int. J. Control 73, 144-151 (2000)

10. Mahmudov, NI: Approximate controllability of semilinear deterministic and stochastic evolution equations in abstract spaces. SIAM J. Control Optim. 42, 1604-1622 (2003)

11. Mahmudov, Nl: Approximate controllability of evolution systems with nonlocal conditions. Nonlinear Anal. 68 , 536-546 (2008)

12. Mahmudov, NI: Controllability of linear stochastic systems in Hilbert spaces. J. Math. Anal. Appl. 259, 64-82 (2001)

13. Benchohra, $\mathrm{M}$, Ouahab, A: Controllability results for functional semilinear differential inclusions in Frechet spaces. Nonlinear Anal. TMA 61, 405-423 (2005)

14. Byszewski, L: Theorems about the existence and uniqueness of solutions of a semilinear evolution nonlocal Cauchy problem. J. Math. Anal. Appl. 162, 494-505 (1991)

15. Górniewicz, L, Ntouyas, SK, O'Regan, D: Controllability of semilinear differential equations and inclusions via semigroup theory in Banach spaces. Rep. Math. Phys. 56, 437-470 (2005)

16. Tai, Z, Wang, X: Controllability of fractional-order impulsive neutral functional infinite delay integrodifferential systems in Banach spaces. Appl. Math. Lett. 22, 1760-1765 (2009)

17. Fu, X: Controllability of non-densely defined functional differential systems in abstract space. Appl. Math. Lett. 19, 369-377 (2006)

18. Fu, X, Mei, K: Approximate controllability of semilinear partial functional differential systems. J. Dyn. Control Syst. 15 425-443 (2009)

19. Klamka, J: Constrained approximate controllability. IEEE Trans. Autom. Control 45, 1745-1749 (2000)

20. Klamka, J: Constrained controllability of semilinear systems with delays. Nonlinear Dyn. 56, 169-177 (2009)

21. Liu, H, Chang, J: Existence for a class of partial differential equations with nonlocal conditions. Nonlinear Anal. 70 , 3076-3083 (2009)

22. Lakshmikantham, V: Theory of fractional differential equations. Nonlinear Anal., Theory Methods Appl. 60(10), 3337-3343 (2008)

23. Mophou, GM, N'Guérékata, GM: Existence of mild solution for some fractional differential equations with nonlocal conditions. Semigroup Forum 79(2), 322-335 (2009)

24. N'Guérékata, GM: A Cauchy problem for some fractional abstract differential equation with nonlocal conditions. Nonlinear Anal. TMA 70(5), 1873-1876 (2009)

25. Ren, Y, Hu, L, Sakthivel, R: Controllability of impulsive neutral stochastic functional differential inclusions with infinite delay. J. Comput. Appl. Math. 235, 2603-2614 (2011)

26. Sakthivel, R, Ren, Y, Mahmudov, NI: Approximate controllability of second-order stochastic differential equations with impulsive effects. Mod. Phys. Lett. B 24, 1559-1572 (2010)

27. Sakthivel, R, Suganya, S, Anthoni, SM: Approximate controllability of fractional stochastic evolution equations. Comput. Math. Appl. 63(3), 660-668 (2012)

28. Sakthivel, R, Nieto, JJ, Mahmudov, Nl: Approximate controllability of nonlinear deterministic and stochastic systems with unbounded delay. Taiwan. J. Math. 14, 1777-1797 (2010)

29. Balachandran, K, Sakthivel, R: Controllability of functional semilinear integrodifferential systems in Banach spaces. J. Math. Anal. Appl. 255, 447-457 (2001)

30. Balachandran, K, Sakthivel, R, Dauer, JP: Controllability of neutral functional integrodifferential systems in Banach spaces. Comput. Math. Appl. 39, 117-126 (2000)

31. Sakthivel, R, Ren, Y, Mahmudov, NI: On the approximate controllability of semilinear fractional differential systems. Comput. Math. Appl. 62, 1451-1459 (2011)

32. Sakthivel, R, Ren, Y: Complete controllability of stochastic evolution equations with jumps. Rep. Math. Phys. 68, 163-174 (2011)

33. Kumar, S, Sukavanam, N: Approximate controllability of fractional order semilinear systems with bounded delay J. Differ. Equ. 252, 6163-6174 (2012)

34. Sukavanam, N, Kumar, S: Approximate controllability of fractional order semilinear delay systems. J. Optim. Theory Appl. 151, 373-384 (2011)

35. Nieto, JJ: Basic theory for nonresonance impulsive periodic problems of first order. J. Math. Anal. Appl. 205, 423-433 (1997)

36. Hino, Y, Murakami, S, Naito, T: Functional Differential Equations with Infinite Delay. Lecture Notes in Mathematics, vol. 1473. Springer, Berlin (1991)

37. Zhou, Y, Jiao, F: Existence of mild solutions for fractional neutral evolution equations. Comput. Math. Appl. 59, 1063-1077 (2010)

38. Zhou, Y, Jiao, F: Nonlocal Cauchy problem for fractional evolution equations. Nonlinear Anal., Real World Appl. 11, 4465-4475 (2010) 
39. Wang, J, Zhou, Y: A class of fractional evolution equations and optimal controls. Nonlinear Anal., Real World Appl. 12, 262-272 (2011)

40. Wang, J, Fan, Z, Zhou, Y: Nonlocal controllability of semilinear dynamic systems with fractional derivative in Banach spaces. J. Optim. Theory Appl. 154(1), 292-302 (2012)

41. Wang, R-N, Liu, J, Chen, D-H: Abstract fractional integro-differential equations involving nonlocal initial conditions in $\alpha$-norm. Adv. Differ. Equ. 2011, 25 (2011)

42. Pazy, A: Semigroups of Linear Operators and Applications to Partial Differential Equations. Springer, New York (1983)

43. Podlubny, I: Fractional Differential Equations. Math. in Science and Eng., vol. 198. Technical University of Kosice, Slovak Republic (1999)

44. Kilbas, AA, Srivastava, HM, Trujillo, JJ: Theory and Applications of Fractional Differential Equations. North-Holland Mathematics Studies, vol. 204. Elsevier, Amsterdam (2006)

45. Yan, Z: Approximate controllability of partial neutral functional differential systems of fractional order with state-dependent delay. Int. J. Control 85, 1051-1062 (2012)

doi:10.1186/1687-2770-2013-118

Cite this article as: Mahmudov and Zorlu: Approximate controllability of fractional integro-differential equations

involving nonlocal initial conditions. Boundary Value Problems 2013 2013:118.

\section{Submit your manuscript to a SpringerOpen ${ }^{\circ}$ journal and benefit from:}

- Convenient online submission

- Rigorous peer review

- Immediate publication on acceptance

Open access: articles freely available online

High visibility within the field

Retaining the copyright to your article 